\title{
Region stability of linear stochastic discrete systems with time-delays
}

\author{
Gang Li ${ }^{1 *} \mathbb{D}$, Yuxia Gao ${ }^{1}$ and Ming Chen
}

\section{"Correspondence:}

ligangccm@163.com

'College of Mathematics and

Systems Science, Shandong

University of Science and

Technology, Qingdao, P.R. China

\begin{abstract}
In this paper, our aim is to investigate the region stability of stochastic discrete time-delay systems. Firstly, region stabilities of systems are defined by a spectrum operator. Secondly, with the aid of linear matrix inequality, some equivalent conditions and related results for region stabilities of systems are obtained. Finally, the relation between region stabilities and the convergence speed of the trajectories of systems is given.
\end{abstract}

Keywords: Region-stability; Time-delay; Discrete stochastic system

\section{Introduction}

Stability is one of essential concepts in dynamical system theory, which has been considered by the researchers in many fields, such as [1-8], and [9-12]. For a linear system without delays, as is well known, the stability is related to the system matrix root-clustering in subregions of the complex plane. That is, the stability of systems has a close relation with the spectrum placement of the system matrix. For example, the further left the spectrum set of the considered system is located, the faster the system response rate is.

Mean square stability has become a hot issue, which has many important applications in system analysis and designs. Some equivalent conditions for mean square stability of linear stochastic systems have been obtained in terms of generalized algebraic Riccati equation (see [13-17]), or linear matrix inequality (LMI) (see [18-21] and [22-25]), or spectra of some operators (see [26-28]). The spectrum technique is very powerful in system analysis and design, which began with [26] for a stochastic system.

Region stability considered in this paper is related with the mean square stability. Region stability problem is to check whether the spectrum set of deterministic or stochastic systems lies in a given region of the complex plane, which has become a popular research focus in the past decades (see [29-33], and the references therein). Compared with deterministic systems, stochastic systems can describe the complexity of practical problems more accurately. For stochastic systems, a special case of region-stability and regionstabilization, called respectively interval stability and interval stabilization, was studied, and a sufficient condition was given for the interval stabilization of general linear stochastic systems with state and control dependent noises in [29]. In [30], the authors further defined and discussed a class of relative stability and stabilization, which is a more special case of region-stability and region-stabilization of linear stochastic systems. The objective of the present work is to make a further investigation in the region stability of linear

(c) The Author(s) 2019. This article is distributed under the terms of the Creative Commons Attribution 4.0 International License (http://creativecommons.org/licenses/by/4.0/), which permits unrestricted use, distribution, and reproduction in any medium, provided you give appropriate credit to the original author(s) and the source, provide a link to the Creative Commons license, and indicate if changes were made. 
stochastic discrete systems. New results concerning the region stability of linear stochastic discrete systems are developed. By means of LMI, some necessary and sufficient conditions are presented for region-stability with generalized LMI regions. To the best of our knowledge, there were no similar results reported for such dynamical systems up to now.

This paper is organized as follows: In Sect. 2, we introduce some LMI regions and generalized LMI regions. In Sect. 3, we give some definitions of region stability and obtain some equivalent conditions and related results for the region stability of stochastic discrete systems with delays.

For convenience, we adopt the following traditional notations: $\mathbb{S}^{n}$ is the set of all symmetric matrices, whose components may be complex; $\mathbb{R}^{n \times m}$ is the set of all $n \times m$ matrices, whose components are real; $A^{\prime}(\operatorname{ker}(A))$ denotes the transpose (kernel space) of the matrix $A ; A \geq 0(A>0)$ is a positive semidefinite (positive definite) symmetric matrix; $I$ is the identity matrix; $\sigma(L)$ is the spectral set of the operator or matrix $L ; \mathbb{N}_{0}=\{0,1,2, \ldots\}$. Here $\otimes$ denotes Kronecker product operation of matrices (see [34] for details); $\mathbb{R}^{n}$ is the set of all $n$-dimensional vectors, whose components are real. For $x \in \mathbb{R}^{n},\|x\|=\sqrt[2]{x^{\prime} x}$.

\section{LMI regions}

In this paper, we shall discuss the stability of time-delay systems in generalized LMI regions. First of all, we introduce some definitions and related examples in LMI regions and generalized LMI regions.

Definition 2.1 ([35]) A region $D$ in the complex plane is called an LMI region if there exist symmetric matrices $H_{1}$ and $H_{2}$ such that

$$
D=\left\{z \in C \mid H_{1}+z H_{2}+\bar{z} H_{2}^{\prime}<0\right\} .
$$

Here we give two examples of LMI region.

Example 2.1 In the complex plane, the circle region $D(q, r)$, which has center $(q, 0)$ and radius $r$, is an LMI region, where $q$ and $r$ satisfy $|q|+r<1$.

Example 2.2 If $r \in(0,1]$, then the fan-shaped region

$$
D(r, \theta)=\left\{z=x+j y\left|x, y \in \mathbb{R}, \tan \theta<-\frac{|y|}{x},\right| z \mid=\sqrt{x^{2}+y^{2}}<r\right\}
$$

is an LMI region.

Different from an LMI region, we introduce another region, which is defined as follows.

Definition $2.2([33])$ For a symmetric matrix

$$
R=\left(\begin{array}{ll}
R_{1} & R_{2} \\
R_{2}^{\prime} & R_{3}
\end{array}\right)
$$

the region

$$
D_{R}=\left\{z \in C \mid R_{1}+z R_{2}+\bar{z} R_{2}^{\prime}+z \bar{z} R_{3}<0\right\}
$$

in the complex plane is called a generalized LMI region. 
Example 2.3 The ring-shaped region $D(0, \alpha, \beta), 0 \leq \alpha<\beta \leq 1$ is a generalized LMI region. Indeed, the matrix in (3) of Definition 2.2 can be written as

$$
R=\left(\begin{array}{cccc}
-\beta^{2} & 0 & 0 & 0 \\
0 & -\alpha^{2} & 0 & 0 \\
0 & 0 & 1 & 0 \\
0 & 0 & 0 & -1
\end{array}\right)
$$

Remark 2.1 In fact, let $R_{3}=0$ in the matrix of (3). Then the region in (4) becomes the region in (1). That is, LMI region is regarded as a special case of a generalized region. The details about the LMI region and generalized region are found in [35].

\section{Criteria of region-stability}

In this section, we shall investigate the region stability of a kind of linear stochastic discrete systems with time-delays:

$$
\left\{\begin{array}{l}
x(t+1)=\sum_{j=0}^{m}\left[F_{j} x(t-j)+G_{j} x(t-j) \omega(t)\right], \\
x(k)=\varphi(k) \in \mathbb{R}^{n}, \quad k=0,-1, \ldots,-m, t \in \mathbb{N}_{0},
\end{array}\right.
$$

where $x(t) \in \mathbb{R}^{n}$ is a column vector, $F_{j}, G_{j} \in \mathbb{R}^{n \times n}, j=0,1, \ldots, m$ are constant coefficient matrices, $\varphi(k) \in \mathbb{R}^{n}, k \in[-m, 0]$ is a deterministic initial condition, $\left\{w(t), t \in \mathbb{N}_{0}\right\}$ is a scalar Brownian motion defined on a complete probability space $\left(\Omega, \mathcal{F}, \mathcal{F}_{t}, \mu\right)$ with $E[w(t)]=0$ and $E[w(t) w(s)]=\delta_{s, t}$, where $\delta_{s, t}$ is the Kronecker delta with $\mathcal{F}_{t}=\sigma\{w(s) \mid 0 \leq s \leq t\}$. For briefness, system (5) is denoted by $\left[\sum_{j=0}^{m} F_{j}, \sum_{j=0}^{m} G_{j}\right]$.

To study the stability of system (5), first of all, we define two vectors $\bar{x}(t)$ and $\bar{x}(0)$ as follows:

$$
\begin{aligned}
& \bar{x}(t)=\left[x^{\prime}(t), x^{\prime}(t-1), \ldots, x^{\prime}(t-m)\right]^{\prime} \in \mathbb{R}^{n(m+1)}, \\
& \bar{x}(0)=\left[\varphi^{\prime}(0), \varphi^{\prime}(-1), \ldots, \varphi^{\prime}(-m)\right]^{\prime}=\bar{x}_{0} \in \mathbb{R}^{n(m+1)} .
\end{aligned}
$$

So system (5) can become the following equivalent stochastic system:

$$
\left\{\begin{array}{l}
\bar{x}(t+1)=F \bar{x}(t)+G \bar{x}(t) \omega(t), \\
\bar{x}(0)=\bar{x}_{0}, \quad t \in \mathbb{N}_{0}
\end{array}\right.
$$

where

$$
F=\left(\begin{array}{ccccc}
F_{0} & F_{1} & \ldots & F_{m-1} & F_{m} \\
I & 0 & \ldots & 0 & 0 \\
\vdots & \vdots & \ddots & \vdots & \vdots \\
0 & 0 & \ldots & I & 0
\end{array}\right), \quad G=\left(\begin{array}{ccccc}
G_{0} & G_{1} & \ldots & G_{m-1} & G_{m} \\
0 & 0 & \ldots & 0 & 0 \\
\vdots & \vdots & \ddots & \vdots & \vdots \\
0 & 0 & \ldots & 0 & 0
\end{array}\right)
$$

Let $X(t)=E\left[\bar{x}(t) \bar{x}^{\prime}(t)\right]$, and suppose $X(t)$ satisfies

$$
X(t+1)=F X(t) F^{\prime}+G X(t) G^{\prime}
$$


Now we define a general Lyapunov operator as follows:

$$
L_{F, G}: X(t) \in \mathbb{S}^{n(m+1)} \rightarrow F X(t) F^{\prime}+G X(t) G^{\prime} \in \mathbb{S}^{n(m+1)} .
$$

Now we give some definitions of region-stability of stochastic time-delay systems by the spectrum operator $\sigma\left(L_{F, G}\right)$.

Definition 3.1 System (5) is $D_{R}$-stable, if $\sigma\left(L_{F, G}\right) \subset D_{R} \subset D(0,1)$.

Definition 3.2 System (5) is $D(q, r)$-stable, if $\sigma\left(L_{F, G}\right) \subset D(q, r) \subset D(0,1)$.

Definition 3.3 System (5) is $D(r, \theta)$-stable, if $\sigma\left(L_{F, G}\right) \subset D(r, \theta) \subset D(0,1)$.

Definition 3.4 System (5) is $D(0, \alpha, \beta)$-stable, if $\sigma\left(L_{F, G}\right) \subset D(0, \alpha, \beta) \subset D(0,1)$.

Now we give some equivalent conditions of these region stabilities.

Theorem 3.1 The time-delay system (5) is $D_{R}$-stable if and only if there exists a positive matrix $P>0$ such that

$$
\begin{aligned}
R_{1} & \otimes P+R_{2} \otimes(P \Theta(H(n, m), F, G))+R_{2}^{\prime} \otimes\left(\Theta(H(n, m), F, G)^{\prime} P\right) \\
& +R_{3} \otimes\left(\Theta(H(n, m), F, G)^{\prime} P(\Theta(H(n, m), F, G))<0,\right.
\end{aligned}
$$

where $\Theta(H(n, m), F, G)=\left[H(n, m)^{\prime} H(n, m)\right]^{-1} H(n, m)^{\prime}[F \otimes F+G \otimes G] H(n, m)$.

Proof Let $X(t)=E\left[\bar{x}(t) \bar{x}^{\prime}(t)\right]$. Then system (5) can be rewritten as a stochastic system

$$
X(t+1)=F X(t) F^{\prime}+G X(t) G^{\prime}
$$

With the knowledge of Kronecker matrix product, system (8) becomes

$$
\vec{X}(t+1)=\widehat{F} \vec{X}(t)
$$

where $\vec{X}(t)=\left[X_{1,1}(t), \ldots, X_{1, n}(t), \ldots, X_{1, n(m+1)}(t), \ldots, X_{n(m+1), n(m+1)}(t)\right]^{\prime} \in \mathbb{R}^{n^{2}(m+1)^{2}}$ and $\widehat{F}=$ $F \otimes F+G \otimes G$. By the theory of $H$ representation method in [36], there exists a full column rank matrix $H(n, m) \in \mathbb{R}^{n^{2}(m+1)^{2} \times \frac{n(m+1)[n(m+1)+1]}{2}}$ such that $\vec{X}=H(n, m) \widetilde{X}, \widetilde{X} \in \mathbb{R}^{\frac{n(m+1)[n(m+1)+1]}{2}}$, so system (9) is equivalent to the following discrete system:

$$
\widetilde{X}(t+1)=\Theta(H(n, m), F, G) \widetilde{X}(t),
$$

where $\Theta(H(n, m), F, G)=\left[H(n, m)^{\prime} H(n, m)\right]^{-1} H(n, m)^{\prime}[F \otimes F+G \otimes G] H(n, m)$. By Theorem 1 in [33], we immediately obtain the result.

From Eqs. (8) and (9) in Theorem 3.1 and Theorem 1 in [27], the relation between the spectrum of operator $L_{F, G}$ and matrix $\Theta(H(n, m), F, G)$ is obtained as follows.

Corollary 3.1 $\sigma\left(L_{F, G}\right)=\sigma(\Theta(H(n, m), F, G))$. 
Remark 3.1 From Theorem 3.1, we know that the time-delay system (5) is $D_{R}$-stable if and only if a certain LMI is feasible.

Similar to Theorem 3.1, it is easy to draw some results as follows.

Theorem 3.2 System (5) is $D(q, r)$-stable if and only if there exists a positive matrix $P>0$ such that the following inequality holds:

$$
\begin{aligned}
& \left(\begin{array}{cc}
-r & -q \\
-q & -r
\end{array}\right) \otimes P+\left(\begin{array}{ll}
0 & 1 \\
0 & 0
\end{array}\right) \otimes(P \Theta(H(n, m), F, G)) \\
& +\left(\begin{array}{ll}
0 & 1 \\
0 & 0
\end{array}\right)^{\prime} \otimes\left(\Theta(H(n, m), F, G)^{\prime} P\right)<0 .
\end{aligned}
$$

Theorem 3.3 System (5) is $D(r, \theta)$-stable if and only if there exists a positive matrix $P>0$ satisfying the inequality in Theorem 3.2 and the following inequality:

$$
\left(\begin{array}{ll}
M & N \\
N^{\prime} & M
\end{array}\right)<0
$$

where

$$
\begin{aligned}
& M=\sin \theta\left(\Theta(H(n, m), F, G) P+P \Theta(H(n, m), F, G)^{\prime}\right), \\
& N=\cos \theta\left(\Theta(H(n, m), F, G) P-P \Theta(H(n, m), F, G)^{\prime}\right) .
\end{aligned}
$$

Theorem 3.4 System (5) is $D(0, \alpha, \beta)$-stable if and only if there exists a positive matrix $P>0$ satisfying

$$
\left(\begin{array}{cc}
-\beta^{2} & 0 \\
0 & \alpha^{2}
\end{array}\right) \otimes P+\left(\begin{array}{cc}
1 & 0 \\
0 & -1
\end{array}\right) \otimes(\Theta(H(n, m), F, G))^{\prime} P \Theta(H(n, m), F, G)<0 .
$$

Using MATLAB, it is quick to check the above criterion of $D_{R}$-stability with LMI. Now we give an example as follows.

Example 3.1 In the time-delay system $\left[\sum_{j=0}^{m} F_{j}, \sum_{j=0}^{m} G_{j}\right]$, let

$$
F_{0}=-\frac{1}{4}, \quad F_{1}=\frac{1}{8}, \quad G_{0}=\frac{1}{16}, \quad G_{1}=\frac{1}{32} .
$$

Then

$$
F=\left(\begin{array}{cc}
-\frac{1}{4} & \frac{1}{8} \\
1 & 0
\end{array}\right), \quad G=\left(\begin{array}{cc}
-\frac{1}{16} & \frac{1}{32} \\
0 & 0
\end{array}\right) .
$$

Through a simple calculation, we can obtain that

$$
H(1,1)=\left(\begin{array}{ccc}
1 & 0 & 0 \\
0 & 1 & 0 \\
0 & 1 & 0 \\
0 & 0 & 1
\end{array}\right), \quad \Theta(H(1,1), F, G)=\left(\begin{array}{ccc}
0.0664 & -0.0664 & 0.0166 \\
-0.2500 & 0.1250 & 0 \\
1.0000 & 0 & 0
\end{array}\right) .
$$


Given $q=\frac{1}{4}, r=\frac{1}{6}$, using MATLAB, we cannot find a feasible solution of the inequality in Theorem 3.2. That is, system (5) is not $D\left(\frac{1}{4}, \frac{1}{6}\right)$-stable.

Choosing $q=\frac{1}{4}, r=\frac{1}{2}$ and using MATLAB, we can obtain that there exists a positive region matrix

$$
P=\left(\begin{array}{ccc}
29.7165 & 0.8568 & 0.0985 \\
0.8568 & 16.4863 & 2.7158 \\
0.0985 & 2.7158 & 3.2825
\end{array}\right)>0
$$

such that

$$
\left(\begin{array}{cc}
-\frac{1}{6} P & -\frac{1}{4} P+P \Theta(H(1,1), F, G) \\
-\frac{1}{4} P+\Theta(H(1,1), F, G)^{\prime} P & -\frac{1}{6} P
\end{array}\right)<0 .
$$

By Theorem 3.2, the system is $D\left(\frac{1}{4}, \frac{1}{2}\right)$-stable.

We use the LMI toolbox of Matlab and commands for obtaining $P$ as follows:

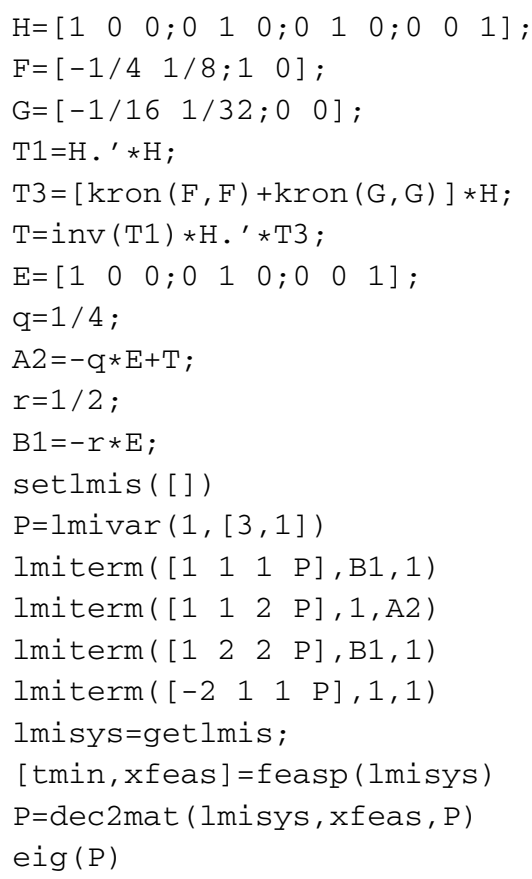

Remark 3.2 Theorems 3.1-3.4 give a kind of characterization for region stability. But the relation between region stabilities and the convergence speed of the trajectories of a system need to be further investigated, which is an interesting and practical problem.

Theorem 3.5 System (5) is $D(0, \alpha, \beta)$-stable if and only if system (1) is such for arbitrary sufficiently small real number $\varepsilon>0$, the convergence speed of the system $\left[\sum_{j=0}^{m} F_{j}, \sum_{j=0}^{m} G_{j}\right]$ is slower than $O\left((\alpha+\varepsilon)^{t}\right)$, but is faster than $O\left((\beta-\varepsilon)^{t}\right)$. That is, there exist two constants $C_{1}>0, C_{2}>0$ such that

$$
C_{2}\left\|\bar{x}_{0}\right\|^{2}(\alpha+\varepsilon)^{t} \leq E\|x(t)\|^{2} \leq C_{1}\left\|\bar{x}_{0}\right\|^{2}(\beta-\varepsilon)^{t} .
$$


Proof (Necessity) Assume that system (5) is $D(0, \alpha, \beta)$-stable. For $\lambda_{i} \in \sigma\left(L_{F, G}\right), i=1,2, \ldots$, $\frac{n(m+1)[n(m+1)+1]}{2}$, without loss of generality, let

$$
\max \left|\lambda_{i}\right|=\left|\lambda_{1}\right|<\beta, \quad \min \left|\lambda_{i}\right|=\left|\lambda_{2}\right|>\alpha .
$$

And let $X(t)=E\left[\bar{x}(t) \bar{x}^{\prime}(t)\right]$, where $\bar{x}(t)=\left[x^{\prime}(t), x^{\prime}(t-1), \ldots, x^{\prime}(t-m)\right]^{\prime} ; x(t)$ is the orbit of system (5) satisfying the initial condition $x(k)=\varphi(k), k=0,-1, \ldots,-m$ (i.e., $\bar{x}_{0}=$ $\left.\left[\varphi^{\prime}(0), \varphi^{\prime}(-1), \ldots, \varphi^{\prime}(-m)\right]^{\prime}\right)$. Using the same proof process as that of Theorem 2.1 in [27], system (5) is equivalent to

$$
X(t+1)=L_{F, G}(X(t)) .
$$

By the theory of $H$ representation method in [36], the initial problem of system (10) is equivalent to the initial problem of the following discrete system:

$$
\left\{\begin{array}{l}
\tilde{X}(t+1)=\Theta(H(n, m), F, G) \widetilde{X}(t), \\
\tilde{X}(0)=\left[H(n, m)^{\prime} H(n, m)\right]^{-1} H(n, m)^{\prime} \vec{X}(0) .
\end{array}\right.
$$

By the stability theory of linear deterministic discrete system, there exist two positive real constants $c_{0}$ and $c^{0}$ such that the following inequality holds:

$$
\begin{aligned}
& \|\tilde{X}(t)\| \leq c_{0}\|\tilde{X}(0)\|\left(\sum_{i=1}^{r_{1}} t^{i-1}\right)\left|\lambda_{1}\right|^{t}, \\
& \|\widetilde{X}(t)\| \geq c^{0}\|\widetilde{X}(0)\|\left(\sum_{i=1}^{r_{2}} t^{i-1}\right)\left|\lambda_{2}\right|^{t},
\end{aligned}
$$

here $r_{1}$ and $r_{2}$ are respectively the multiplicities of the characteristic roots $\lambda_{1}$ and $\lambda_{2}$. For $\left|\lambda_{1}\right|<\beta,\left|\lambda_{2}\right|>\alpha$, by the denseness of real numbers, there exists a sufficiently small number $\varepsilon>0$, such that $\left|\lambda_{1}\right|-\beta+\varepsilon<0,\left|\lambda_{2}\right|-\alpha-\varepsilon>0$, and so, by the results in [29], we can obtain the following inequality:

$$
E\|\bar{x}\|^{2} \leq \sqrt{n(m+1)}\|\widetilde{X}\|, \quad\|\widetilde{X}\| \leq \sqrt{\frac{n(m+1)+1}{2}} E\|\bar{x}\|^{2} .
$$

So

$$
E\|\bar{x}(t)\|^{2} \leq c_{1}\left\|\bar{x}_{0}\right\|^{2}(\beta-\varepsilon)^{t}, \quad E\|\bar{x}(t)\|^{2} \geq c_{2}\left\|\bar{x}_{0}\right\|^{2}(\alpha+\varepsilon)^{t},
$$

where

$$
\begin{aligned}
& c_{1}=c_{0} \sqrt{\frac{n(m+1)[n(m+1)+1]}{2}} \sup _{t \in[0, \infty)}\left(\sum_{i=1}^{r_{1}} t^{i-1}\right)\left(\frac{\left|\lambda_{1}\right|}{\beta-\varepsilon}\right)^{t}<\infty, \\
& c_{2}=c^{0} \sqrt{\frac{2}{n(m+1)[n(m+1)+1]}} .
\end{aligned}
$$


Hence, from the above inequality, we can obtain that there exist two constants $C_{1}>0$, $C_{2}>0$ such that

$$
E\|x(t)\|^{2} \leq C_{1}\left\|\bar{x}_{0}\right\|^{2}(\beta-\varepsilon)^{t}, \quad E\|x(t)\|^{2} \geq C_{2}\left\|\bar{x}_{0}\right\|^{2}(\alpha+\varepsilon)^{t} .
$$

(Sufficiency) Suppose that system (5) is $(\alpha, \beta)$ region stable. For arbitrary real number $\varepsilon>0$, the convergence speed of the system $\left[\sum_{j=0}^{m} F_{j}, \sum_{j=0}^{m} G_{j}\right]$ is slower than $O\left((\alpha+\varepsilon)^{t}\right)$, but is faster than $O\left((\beta-\varepsilon)^{t}\right)$. That is, there exist two constants $C_{1}>0, C_{2}>0$ such that

$$
C_{2}\left\|\bar{x}_{0}\right\|^{2}(\alpha+\varepsilon)^{t} \leq E\|x(t)\|^{2} \leq C_{1}\left\|\bar{x}_{0}\right\|^{2}(\beta-\varepsilon)^{t} .
$$

Note that $\bar{x}(t)=\left[x^{\prime}(t), x^{\prime}(t-1), \ldots, x^{\prime}(t-m)\right]^{\prime}$, and it is easy to obtain that there exist two numbers $c_{1}, c_{2}$ satisfying

$$
c_{2}\left\|\bar{x}_{0}\right\|^{2}(\alpha+\varepsilon)^{t} \leq E\|\bar{x}(t)\|^{2} \leq c_{1}\left\|\bar{x}_{0}\right\|^{2}(\beta-\varepsilon)^{t} .
$$

Note that $\bar{x}(t)$ is the trajectory of system (6), so from (12), we can obtain $\sigma\left(L_{F, G}\right) \subset$ $D(0, \alpha, \beta) \subset D(0,1)$. The proof is completed.

Corollary 3.2 If system (5) is $D_{R}$-stable then there exists a constant $C>0$ such that $\lim _{t \rightarrow+\infty} E\|x(t)\|^{2}=0$.

Corollary 3.3 If system (5) is $D(q, r)$-stable then there exist two constants $C_{1}>0, C_{2}>0$ such that $C_{2}\left\|\bar{x}_{0}\right\|^{2}(\|q\|+\varepsilon)^{t} \leq E\|x(t)\|^{2} \leq C_{1}\left\|\bar{x}_{0}\right\|^{2}((\|q\|+r)-\varepsilon)^{t}$.

Corollary 3.4 If system (5) is $D(r, \theta)$-stable then there exists a constant $C>0$ such that $E\|x(t)\|^{2} \leq C\left\|\bar{x}_{0}\right\|^{2}(r-\varepsilon)^{t}$.

Remark 3.3 From Theorem 3.5 and Corollaries 3.2-3.4, region stabilities defined in this paper imply mean square stability of system (5).

\section{Conclusion}

In this paper, we investigated the region stability of discrete stochastic time-delay systems. First of all, we defined the notions of region-stability of discrete stochastic timedelay systems. We then gave some equivalent conditions for the region stabilization of linear stochastic discrete time-delay systems. We only considered the stability of discrete stochastic time-delay systems on some LMI regions. But the stability of discrete stochastic time-delay systems on general regions is still a challenging problem.

\footnotetext{
Acknowledgements

The authors wish to thank editor and anonymous reviewer for his/her valuable suggestions to this paper.

Funding

This work was supported by the National Natural Science Foundation of China under Grants 61503224, National Science Foundation of Shandong Province ZR2017MF054, Qingdao Postdoctoral Applied Research Project No. 2015188 and SDUST Research Fund No. 2015TDJH105.
} 


\section{Competing interests}

All the authors declare that there is no conflict of interest regarding the publication of this paper.

\section{Authors' contributions}

All authors contributed equally to the writing of this paper. All authors read and approved the final paper.

\section{Publisher's Note}

Springer Nature remains neutral with regard to jurisdictional claims in published maps and institutional affiliations.

Received: 26 February 2019 Accepted: 2 July 2019 Published online: 13 August 2019

\section{References}

1. Mao, X:: Stochastic Differential Equations and Their Applications. Horwood, Chichester (1997)

2. Coddington, E.A., Levinson, N.: Theory of Ordinary Differential Equations. McGraw-Hill, New York (1955)

3. Has'minskii, R.Z.: Stochastic Stability of Differential Equations. Sijthoff \& Noordhoff, Alphen (1980)

4. Kushner, H.J.: Stochastic Stability and Control. Academic Press, New York (1967)

5. Chen, M., Xia, D., Wang, D., Han, J., Liu, Z.: An analytical method for reducing metal artifacts in X-ray CT images. Math. Probl. Eng. 2019, Article ID 2351878 (2019)

6. Li, G., Chen, M.: Intertwined phenomenon of a kind of dynamical system. Adv. Differ. Equ. 2013(1), Article ID 265 (2013)

7. Li, G., Ding, C., Chen, M.: Intertwined basins of attraction of dynamical systems. Appl. Math. Comput. 213(1), 272-274 (2009)

8. Zhang, T., Meng, X., Zhang, T.: Global analysis for a delayed SIV model with direct and environmental transmissions. J. Appl. Anal. Comput. 6(2), 479-491 (2016)

9. Baleanu, D., Wu, G., Bai, Y., Chen, F.: Stability analysis of Caputo-like discrete fractional systems. Commun. Nonlinear Sci. Numer. Simul. 48, 520-530 (2017)

10. Wu, G., Baleanu, D., Zeng, S.: Finite-time stability of discrete fractional delay systems: Gronwall inequality and stability criterion. Commun. Nonlinear Sci. Numer. Simul. 57, 299-308 (2018)

11. Wu, G., Baleanu, D., Zeng, S.: Several fractional differences and their applications to discrete maps. J. Appl. Nonlinear Dyn. 4, 339-348 (2015)

12. Li, G., Gao, Y., Chen, M.: Attractors of dynamical systems in locally compact spaces. Open Math. 17, 465-471 (2019)

13. Li, G., Chen, M.: On uniqueness of strong solution of stochastic systems. Abstr. Appl. Anal. 2014, Article ID 890925 (2014)

14. Gao, M., Sheng, L., Zhang, W.: Stochastic $\mathrm{H}_{2} / \mathrm{H}_{\infty}$ control of nonlinear systems with time-delay and state-dependent noise. Appl. Math. Comput. 266, 429-440 (2015)

15. Liu, X., Li, Y., Zhang, W.: Stochastic linear quadratic optimal control with constraint for discrete-time systems. Appl. Math. Comput. 228, 264-270 (2014)

16. Zhang, W., Zhang, H., Chen, B.S.: Generalized Lyapunov equation approach to state-dependent stochastic stabilization/detectability criterion. IEEE Trans. Autom. Control 53, 1630-1642 (2008)

17. Li, G., Zhang, W., Chen, M.: Robust $\mathrm{H}_{2} / \mathrm{H}_{\infty}$ control for periodic stochastic difference systems with multiplicative noise. IET Control Theory Appl. 9(16), 2451-2457 (2015)

18. Yan, Z., Zhang, G., Wang, J., Zhang, W.: State and output feedback finite-time guaranteed cost control of linear ito stochastic systems. J. Syst. Sci. Complex. 28, 813-829 (2015)

19. Boyd, S., El Ghaoui, L., Feron, E., Balakrishnan, V.: Linear Matrix Inequalities in System and Control Theory. SIAM, Philadelphia (1994)

20. Ait Rami, M., Zhou, X.Y.: Linear matrix inequalities, Riccati equations and indefinite stochastic linear quadratic control. IEEE Trans. Autom. Control 45, 1131-1142 (2000)

21. Boyd, S., Ghaoui, E., Feron, E., Balakrishnan, V.: Linear Matrix Inequalities in System and Control Theory. SIAM, Philadelphia (1994)

22. Mobayen, S., Baleanu, D.: Linear matrix inequalities design approach for robust stabilization of uncertain nonlinear systems with perturbation based on optimally-tuned global sliding mode control. J. Vib. Control 23, 1285-1295 (2017)

23. Faieghi, M.R., Kuntanapreeda, S., Delavari, H., Baleanu, D.: Robust stabilization of fractional-order chaotic systems with linear controllers: LMI-based sufficient conditions. J. Vib. Control 20, 1042-1051 (2014)

24. Mobayen, S., Baleanu, D., Tchier, F.: Second-order fast terminal sliding mode control design based on LMI for a class of non-linear uncertain systems and its application to chaotic systems. J. Vib. Control 23, 2912-2925 (2016)

25. Li, G., Chen, M.: Infinite horizon linear quadratic optimal control for stochastic difference time-delay systems. Adv. Differ. Equ. 2015, Article ID 14 (2015)

26. Zhang, W., Chen, B.S.: On stabilizability and exact observability of stochastic systems with their applications. Automatica 40, 87-94 (2004)

27. Li, G., Chen, M.: The stability and stabilization of stochastic delay-time systems. Math. Probl. Eng. 2014, Article ID 272745 (2014)

28. Hou, T., Ma, H., Zhang, W.: Spectral tests for observability and detectability of periodic Markov jump systems with nonhomogeneous Markov chain. Automatica 63, 175-181 (2016)

29. Zhang, W., Xie, L.: Interval stability and stabilization of linear stochastic systems. IEEE Trans. Autom. Control 54 810-815 (2009)

30. Zhang, W.: General D-stability and D-stabilization for linear stochastic systems: continuous-time case. In: 2010 8th IEEE International Conference on Control and Automation (2010)

31. Fragoso, M.D., Costa, O.L.V., de Souza, C.E.: A new approach to linearly perturbed Riccati equations arising in stochastic control. Appl. Math. Optim. 37, 99-126 (1998) 
32. El Harraki, I., El Alami, A., Boutoulout, A., Serhani, M.: Regional stabilization of semi-linear parabolic systems. IMA J. Math. Control Inf. 34, 961-971 (2017)

33. Peaucelle, D., Arzelier, D., Bachelier, O., Bernussou, J.R.K.: A new robust D-stability condition for real convex polytypic uncertainty. Syst. Control Lett. 40, 21-30 (2000)

34. Ortega, J.M.: Matrix Theory. Plenum, New York (1987)

35. Chilali, M., Gahinet, P.: $H_{\infty}$ design with pole placement constraints: an LMI approach. IEEE Trans. Autom. Control 41 , 358-367 (1996)

36. Zhang, W., Chen, B.S.: H-representation and applications to generalized Lyapunov equations and linear stochastic systems. IEEE Trans. Autom. Control 57, 3009-3022 (2012)

Submit your manuscript to a SpringerOpen ${ }^{\circ}$ journal and benefit from:

- Convenient online submission

Rigorous peer review

Open access: articles freely available online

- High visibility within the field

- Retaining the copyright to your article

Submit your next manuscript at $\boldsymbol{~ s p r i n g e r o p e n . c o m ~}$ 